\title{
Application Research of Zhengyou Zhang Calibration Method in Visual Recognition of Hull Welds
}

\author{
Lei Ou, Xiaobo Gu
}

\begin{abstract}
In the application of the hull weld visual recognition system, the camera in the vision system was calibrated based on the Zhengyou Zhang calibration method, and the calibration method was improved. The camera calibration with Matlab as the tool was given. In the steps, the radial distortion and tangential distortion generated during the calibration process were corrected and analyzed, and the error of camera calibration results on the visual identification of the final weld was analyzed. The calibration results show that the calibration method can ensure the accuracy of the camera calibration and the process is simple. It can meet the accuracy requirements of camera calibration in the visual identification of hull welds, and provides an experimental basis for the application of Zhengyou Zhang calibration method in the visual identification of hull welds.
\end{abstract}

Index Terms - hull weld, visual recognition, calibration method of zhengyou zhang

\section{INTRODUCTION}

In the process of transforming the modern shipbuilding mode into the intelligent shipbuilding mode, the automation of ship welding is one of the important manifestations of the transformation of shipbuilding mode. The working hours and costs of hull welding account for about $30 \%$ to $40 \%$ of the total working hours and total cost of the whole ship[1],Promoting the automation and intelligence of welding is the key link to promote intelligent manufacturing of ships. Some shipyards have realized the robotic welding automation of the teaching reproduction mode in the welding of the panel, the T-profile and the ball flat steel, and the partial group welding, which has improved the welding efficiency of the ship to some extent. The application of machine vision recognition can realize the high degree of automation of the industry in a deeper level.

It is widely used and matured in the industries of rubber coating, grabbing and automobile assembly. The hull weld visual recognition system combines machine vision and welding robot in hull workpiece.Among the weld seams, it can effectively simplify the cumbersome process of teaching and effectively improve the precision and efficiency of welding automation. The calibration accuracy of the camera directly affects the subsequent hull weld identification accuracy of the entire hull weld visual recognition system. Therefore, based on the Zhengyou Zhang calibration method, the camera is calibrated using the Matlab camera calibration toolbox and generated during the calibration process. The distortion and error were analyzed and corrected. Finally, the

Lei Ou,Xiaobo Gu, School of Naval Architecture \& Ocean ngineering, Jiangsu University of Science and Technology, Zhenjiang, Jiangsu, China influence of the calibration results on the actual weld identification is analyzed, and the accuracy and reliability of the selected camera in the hull weld identification are verified.

\section{VISION SYSTEM CALIBRATION METHOD}

There are three classic calibration methods in the traditional method of machine vision camera calibration, which are the traditional photogrammetry calibration method, self-calibration method and active visual calibration method [2]. The Zhengyou Zhang calibration method integrates the advantages of the traditional measurement calibration method and the self-calibration method, and is a calibration method between the two calibration methods [3]. The Zhengyou Zhang calibration method is based on two or more calibrations of the chessboard image for calibration experiments, and can be ingested by the camera fixed, the chessboard fixed, and the camera and the chessboard simultaneously moving when the image of the checkerboard is ingested. The internal and external parameters of the camera are obtained by a certain calibration algorithm. The method not only reduces the expensive experiment cost of the traditional photogrammetry calibration method but also simplifies the experimental steps, and at the same time makes up for the problem of insufficient precision of the self-calibration method in the calibration process. Compared with the above two calibration methods, the robustness is obtained. Improvement, calibration accuracy has also been effectively improved.

Zhengyou Zhang calibration method has maintained the accuracy of calibration while ensuring the simplicity of the calibration experiment. It has become one of the main methods for camera calibration of machine vision systems. In this paper, Zhengyou Zhang calibration method is used to improve the adaptation requirements: 1: The square lattice calibration plate used by it is changed to black and white chessboard, which makes the corner points more continuous and easy to detect;2: When the visual camera is calibrated, a small number of calibration plate images can no longer meet the calibration accuracy requirements, and the number of experimental calibration chessboards is set to 20 to ensure the accuracy of the calibration.

\section{A. Camera calibration principle}

The camera calibration principle in the vision system is based on the principle of small hole imaging. The principle model is shown in Figure 1. The $\mathrm{f}$ and $\mathrm{z}$ in the figure are the focal length and the object distance respectively. Most industrial CCD cameras on the market are composed of lenses, and their imaging mode is similar to the principle of small hole imaging. In order to complete this calibration, through the 
demand analysis and calculation, this paper selects MV-GED200M-T industrial camera with resolution of $1600 \times 1200$ and MV-GED200M-T camera lens with focal length of $8 \mathrm{~mm}$ as the visual part of hull weld visual recognition system.

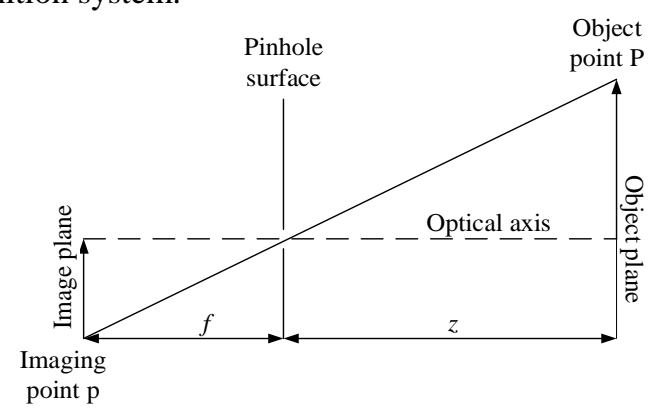

Fig 1 Small hole imaging schematic

The world coordinate system $\left(\mathrm{X}_{\mathrm{W}}, \mathrm{Y}_{\mathrm{W}}, \mathrm{Z}_{\mathrm{W}}\right)$, the camera coordinate system $\left(\mathrm{X}_{\mathrm{C}}, \mathrm{Y}_{\mathrm{C}}, \mathrm{Z}_{\mathrm{C}}\right)$, the image coordinate system $(\mathrm{x}, \mathrm{y})$, and the pixel coordinate system $(\mathrm{u}, \mathrm{v})$ are the four main coordinate systems in the visual recognition system. The conversion relationship between them is shown in Figure 2. The conversion between the world coordinate system and the camera coordinate system can be represented by the rotation matrix $\mathbf{R}$ and the translation vector $\boldsymbol{\tau}$, and the rotation matrix and the translation vector can be calculated by camera calibration.

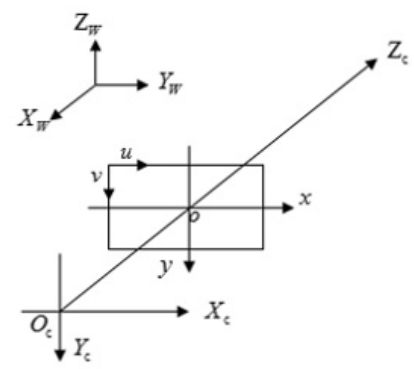

Fig 2 Relationship between four coordinate systems during imaging

The relationship between a point $\mathrm{p}$ and the world coordinate system in the camera coordinate system can be represented by a rotation matrix and a translation vector[4], as shown in equation (1).

$$
\left[\begin{array}{c}
x_{p} \\
y_{p} \\
z_{p} \\
1
\end{array}\right]=\left[\begin{array}{cc}
R & t \\
0_{1 \times a} & 1
\end{array}\right]\left[\begin{array}{c}
X_{W} \\
Y_{W} \\
Z_{W} \\
1
\end{array}\right]=M_{2}\left[\begin{array}{c}
X_{W} \\
Y_{W} \\
Z_{W} \\
1
\end{array}\right]
$$

In actual use of the vision system camera, due to the manufacturing process of the camera and the optical lens lens and the error caused by the assembly of the entire vision system, the distortion of the camera when the image is captured may affect the imaging effect of the image. Radial and tangential distortions in the acquisition process of the calibration chessboard image have a relatively large influence on the image acquisition results. Therefore, the effects of these two distortions are analyzed and corrected to correct the coordinates before and after the radial distortion. The point conversion relationship is as shown in equation (2):

$$
\left\{\begin{array}{l}
x_{\text {rcorr }}=x_{p}\left(1+k_{1} r^{2}+k_{2} r^{4}+k_{a} r^{6}\right) \\
y_{\text {rcorr }}=y_{p}\left(1+k_{1} r^{2}+k_{2} r^{4}+k_{a} r^{6}\right)
\end{array}\right.
$$

Correcting the conversion relationship of coordinate points in the coordinate system before and after tangential distortion is shown in equation (3) :

$$
\left\{\begin{array}{l}
x_{\text {tcorr }}=x_{p}+\left[2 p_{1} x_{p} y_{p}+p_{2}\left(r^{2}+2 x_{p}^{2}\right)\right] \\
y_{\text {tcory }}=y_{p}+\left[2 p_{2} x_{p} y_{p}+p_{1}\left(r^{2}+2 y_{p}^{2}\right)\right]
\end{array}\right.
$$

The demand solves the $\mathrm{k}_{1}, \mathrm{k}_{2}, \mathrm{k}_{3}, \mathrm{p}_{1}$, and $\mathrm{p}_{2}$ in equations (2) and (3) to calculate the coordinate values after correcting radial distortion and tangential distortion. The five unknown coefficients $\mathrm{k}_{1}, \mathrm{k}_{2}, \mathrm{k}_{3}, \mathrm{p}_{1}, \mathrm{p}_{2}$ in (2)and (3) can be obtained by camera calibration, which can effectively correct the influence of distortion on the imaging effect.

\section{B. Matlab calibration steps}

Before the camera calibration, the calibration chessboard should be designed and drawn. The number of grids in the horizontal and vertical directions of the calibration chessboard used in this calibration is $7 \times 9$, and the actual size of the black and white grid is $28 \mathrm{~mm} \times 28 \mathrm{~mm}$. The : 1 ratio is printed out, placed on a flat plane for camera shooting to extract the image of the calibration chessboard, and the black and white calibration chessboard is shown in Figure 3.

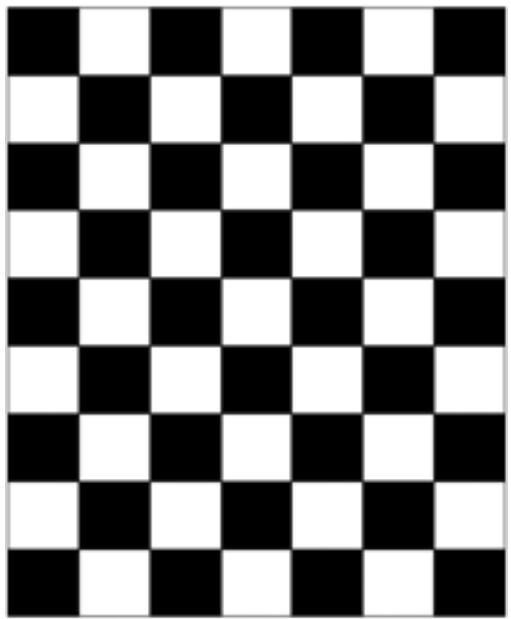

Fig 3 Black and white calibration chessboard

In the process of collecting the image of the chessboard, the image of the calibration chessboard is collected by the method of fixing the chessboard and moving the camera. The camera is fixedly fixed to the distance of $70 \mathrm{~cm}$ from the chessboard. The converted shooting angle is taken by 20 calibration board images. The 20 images are shown in Figure 4.

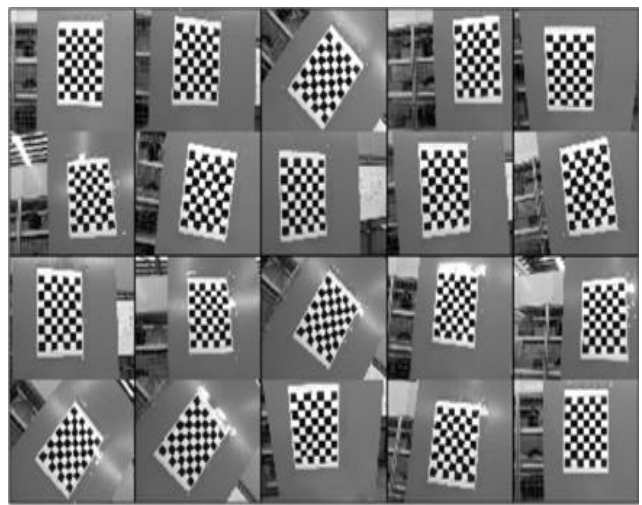

Fig 4 Calibration board image 
The detailed camera calibration steps in Matlab are as follows

1 ) Calibration of the calibration image: Before the calibration image is read, the naming prefixes of the 20 images are unified. After naming the prefix, the images are distinguished by numbers, and the suffix format type of each image is also unified. The experimental image naming format is img1.jpg. And the image file needs to be placed in the same folder as the camera calibration module. After selecting the image type, the image of the chessboard image is read one by one.

2) Corner extraction: Corner extraction is performed on a pair of 20 images, and the selected black and white chessboard area is selected in a counterclockwise four-point manner. Fig of 5 is a corner extraction map of the calibration image img1.jpg. For example, after the corner point is extracted, the corner point is located within the chessboard of the calibration chessboard image, indicating that the corner point extraction fails, and the appeal step is repeated to perform the corner point extraction operation on the image again.

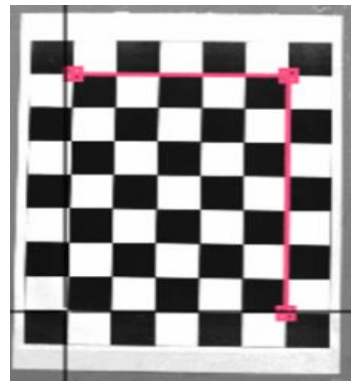

(a)Corner extraction order

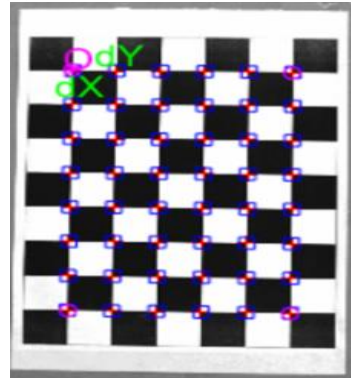

(b) Corner extraction succeeded

Fig 5 Corner extraction

3) After the corner points are extracted, the internal parameters of the camera are calibrated, and the relationship between the camera and the calibration chessboard is displayed in the form of a graph, and the re-projection error distribution of the image space is calculated.

4) The corner image of the calibration chessboard image is extracted again and the external parameters of the camera are calculated. Finally, the calibration result data is saved.

\section{CALIBRATION RESULT ANALYSIS}

The visual identification of ship welding path in this paper is still in the laboratory stage. It verifies the feasibility of applying Zhang Zhengyou's calibration method in the hull welding path identification system, and verifies the accuracy of the camera in the hull weld identification in the visual recognition system. Provide experimental references for ship welding applications.
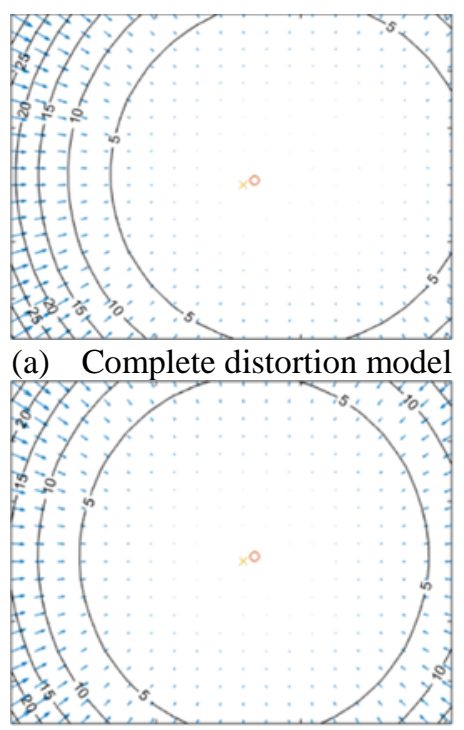

(b) Radial distortion model



(c) Tangential distortion model

Fig 6 The effect of distortion on image pixels

Figure 6 shows the effect of radial distortion on the image pixels during the calibration process. It can be seen from Fig. a) that the complete distortion model has the greatest influence on the image pixels, and the pixel value is shifted by 25 values. The main reason is the pixel value offset caused by distortion, the maximum influence of tangential distortion on the pixel value is 8 , compared to the radial distortion tangential distortion has a relatively small impact on the overall distortion model.

Figure 7 shows the contrast analysis of the pixel re-projection error in the camera calibration before and after the distortion correction. The re-projection error refers to the deviation of each corner point of each image during the extraction of the corner point during the calibration process, as shown in the figure. The deviation of each detection corner can determine the accuracy and reliability of the camera calibration result. The pixel errors in the $\mathrm{x}$ and $\mathrm{y}$ directions of the pixel shown in Figure a) are within the range of $[-0.6,0.6]$ and $[-0.4,0.5]$, respectively, and the pixel errors in the $\mathrm{x}$ and $\mathrm{y}$ directions after correction are $[-0.5,0.4]$ within the range of $[-0.3,0.4]$, the pixel accuracy is improved compared to the correction. In [5], it is pointed out that the re-projection error is about 2 pixels, and the accuracy of the camera calibration is relatively high, which can meet the requirements of camera calibration. The re-projection error of this camera calibration is maintained within 0.5 pixels, which is enough to meet the requirements of camera calibration, and indicates that the calibration accuracy is relatively high. 


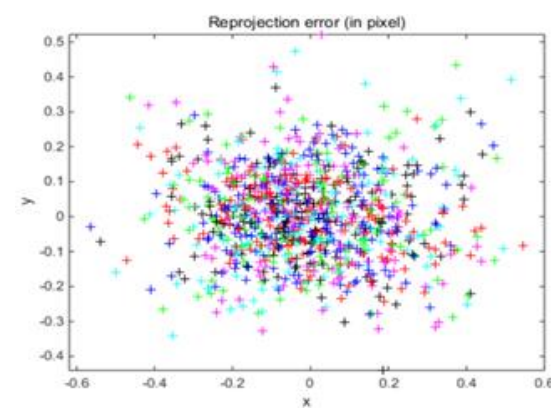

(a) Corrected pre-projection error

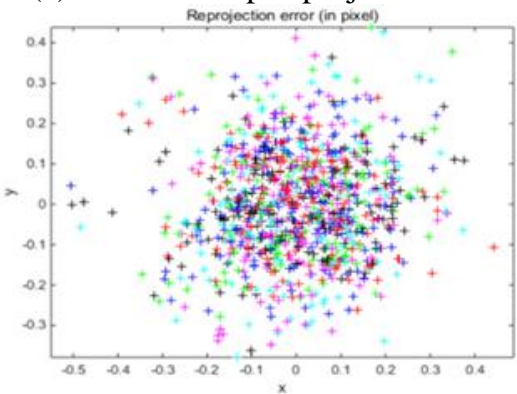

(b) Corrected reprojection error

Fig 7 Comparative analysis of re-projection errors before and after correction

In order to more clearly show the positional relationship between the camera and the calibration chessboard, Matlab is used to compare the relationship between the two in the calibration, as shown in Figure 8, the camera shown in the figure and the calibration chessboard. The relative distance is basically the same as the actual distance when the image is taken, which further verifies the reliability of the camera calibration.



(a) Camera fixed

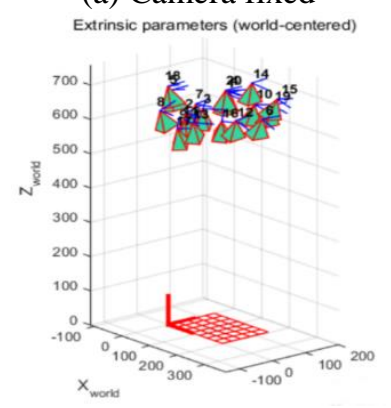

(c) Calibration plate fixed

Fig 8 Relative positional relationship between the camera and the calibration chessboard

The rotation matrix and translation vector results obtained by camera calibration are shown in equations (4) and (5). The calculation results of the five unknown distortion coefficients in the correction distortion process are as shown in equation (6).

$$
\begin{aligned}
& R=\left[\begin{array}{ccc}
-0.01404 & 0.99989 & -0.00459 \\
0.99921 & 0.01387 & -0.03704 \\
-0.03697 & -0.00511 & -0.99930
\end{array}\right] \\
& \tau=\left[\begin{array}{lll}
-45.22453 & -114.06011 & 707.13803
\end{array}\right](5) \\
& \left(\mathrm{k}_{1} \cdot \mathrm{k}_{2} \cdot \mathrm{k}_{3} 、 \mathrm{p}_{1} \cdot \mathrm{p}_{2}\right)=(-0.07882,-0.08566,-0.00118 \\
& , 0.00598,0.00000)(6)
\end{aligned}
$$

\section{WELDING SEAM VISUAL RECOGNITION ERROR ANALYSIS}

In order to verify whether the error accuracy of the calibration result meets the requirements of the hull weld visual recognition experiment, the workpiece image visually recognized by the hull weld is image-processed based on the completion of the camera calibration. The welding figure original drawing, image edge processing and weld center line extraction result comparison chart are shown in Fig. 9.



(a) Identify the original artwork

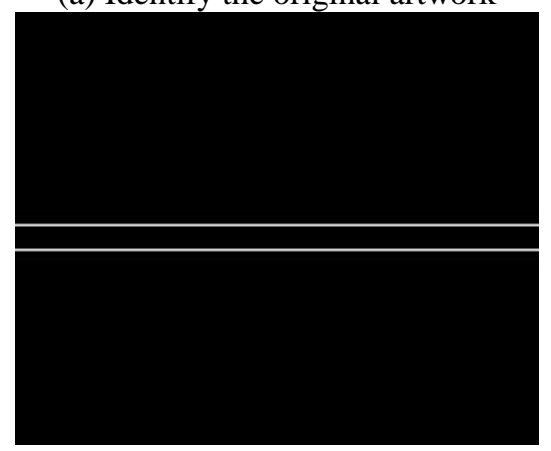

(b) Visual recognition effect map

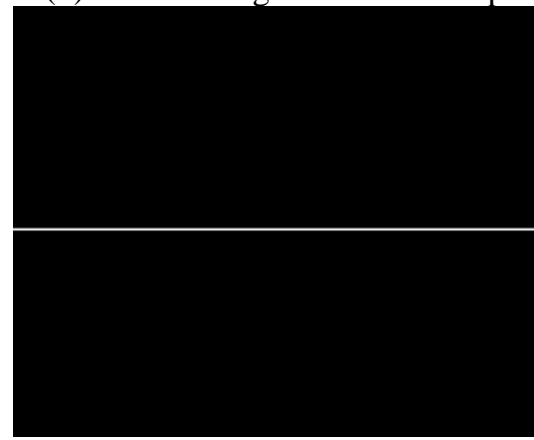

(c) Weld centerline image

Fig 9 Weld visual recognition effect map

The average width of the pixel width is 13.1 pixels wide by 20 times of manual identification of the workpiece width of the workpiece image, and the workpiece weld width pixel value automatically recognized by the image processing by the average method is 13.5 pixels wide. The average error in 
identifying the weld pixel width is 0.4 pixel values. The camera re-projection error obtained by improving the Zhengyou Zhang calibration method is basically maintained within 0.5 pixels, and the pixel error of the entire visual recognition system is within 0.9 pixel values. The actual width of the weld of the visually recognized workpiece is $15 \mathrm{~mm}$, and the actual weld width error is within $1 \mathrm{~mm}$ by the actual scale conversion. The weld seam identifies the actual width error to meet the error range requirement of the actual weld width.

\section{CONCLUSION}

The re-projection error obtained by camera calibration is kept within 0.5 pixels. The actual identification error of the weld width of the whole visual recognition system is kept within $1 \mathrm{~mm}$, which indicates that the application of Zhengyou Zhang calibration method can be sufficient in the hull weld visual recognition system. The accuracy and calibration test verify that about 20 calibration board images are enough 'to meet the accuracy of camera calibration, and demonstrate the feasibility of Zhengyou Zhang calibration method in the hull weld visual recognition system. In this experiment, the effects of radial distortion and tangential distortion on the experiment were taken into account and corrected, which effectively improved the accuracy of camera calibration. However, due to the workpiece the influence of the error caused by the distortion generated when the image is spliced.

\section{REFERENCE}

[1] ZHANG Wei,FU Dao,YAN Dao-hai,LI Hong-xi.Analysis and Countermeasures of the Development Status of Shipbuilding Technology[J]. Ship Science and Technology,2013,35(02):9-12.

[2] Zhang Z . A Flexible New Technique for Camera Calibration[M]. IEEE Computer Society, 2000.

[3] Liu Lin, Chen Xiang, Liu Juanxiu, et al. Two-dimensional photoelectric image measuring instrument system camera calibration technology [J]. China Science and Technology Information, 2016(11): 20 23

[4] Zhao Peng. Research and Development of Machine Vision[M]. Science Press, 2012.

[5] Fan Fang, Wang Zhenwei, Liu Shuangyin, et al. Calibration Error Analysis of Cameras for Visual Measurement[J]. Experiment Science And Technology, 2016, 14(6): 14-16.

Lei Ou, School of Naval Architecture \& Ocean Engineering, Jiangsu University of Science and Technology, Zhenjiang, Jiangsu, China

Xiaobo Gu, Professor, School of Naval Architecture \& Ocean Engineering, Jiangsu University of Science and Technology, Zhenjiang, Jiangsu, China 